\title{
Bivalence and Future Contingency
}

\section{Sandu, Gabriel}

Springer

2018

Sandu , G , Carlo , P \& Rivenc , F 2018 , Bivalence and Future Contingency . in S O

Hansson \& V Hendricks (eds), Introduction to Formal Philosophy . Springer Undergraduate

Texts in Philosophy, Springer, Switzeralnd, pp. 333-350 .<

https://link.springer.com/chapter/10.1007/978-3-319-77434-3_16 >

http://hdl.handle.net/10138/320592

unspecified

acceptedVersion

Downloaded from Helda, University of Helsinki institutional repository.

This is an electronic reprint of the original article.

This reprint may differ from the original in pagination and typographic detail.

Please cite the original version. 


\title{
Bivalence and future contingency
}

\author{
Gabriel Sandu, Carlo Proietti, François Rivenc
}

\begin{abstract}
This work presents an overview of four different approaches to the problem of future contingency and determinism in temporal logics. All of them are bivalent, viz. they share the assumption that propositions concerning future contingent facts have a determinate truth-value (true or false). We introduce Ockhamism, Peirceanism, Actualism and $T \times W$ semantics, the four most relevant bivalent alternatives in this area, and compare them from the point of view of their expressiveness and their underlying metaphysics of time.
\end{abstract}

Keywords : Determinism, Temporal Logics, Future Contingents, Actualism, Ockhamism, Peirceanism, $T \times W$.

\section{Introduction}

A major problem for schoolmen was to reconcile divine foreknowledge with future contingency, this last being a prerequisite for human free choice. In modern times when theological concerns are less pressing, the so-called future contingents problem has shifted back to the more mundane Aristotelian question of how to accommodate the latter with the principle of bivalence, i.e. the thesis that all propositions, including those concerning future contingent facts, are either true or false. Both problems amount to the same if one assumes that only true propositions may be known (nihil scitum nisi verum) and that God has a full science about the future. But if one doesn't care much about God's omniscience, then this puzzle becomes less urgent and one may just solve the dilemma by discarding one of its horns, i.e. the principle of bivalence. This is what most of the contemporary approaches to future contingency do (see Belnap and Green (1994), Thomason (1970), MacFarlane (2003)). ${ }^{1}$

\footnotetext{
${ }^{1}$ The forerunner of all these solutions has been considered by many scholars (but not all of them) to be Aristotle in chapter IX of On Interpretation.
} 
Nonetheless, there are many reasons for preserving bivalence. Logical simplicity is perhaps the most instrumental of them. Others are related to language expressivity and the fact that non bivalent approaches seem mostly unable to distinguish simple truths about the future from settled truths about it.

There are several bivalence-preserving solutions to the future contingents problem. Many of them were already known to the scholastics (see Øhrstrom (2009)). We will present those which have preserved their relevance up to nowadays: Ockhamism, Peirceanism (both formulated by Prior), actualism and $W \times T$ semantics. All of them (except maybe Peirceanism) have been inspired by the medieval tradition. The advantage of contemporary tensedlogical approaches lies in their rigor and their comparability, mostly due to the fact that they all have the same semantic format.

There are no shared desiderata for a best choice among these solutions. Metaphysical considerations, tacit or explicit, about the "real" structure of time play a major role in the discussion and may easily turn into an "ideological" debate. Nonetheless, it is instructive to compare how the different approaches account not only for the openness of the future, but also for some additional intuitions about time and truth. One of these is retrogradation of truth. When one evaluates ex post a sentence like "there will be a sea-battle tomorrow" she is driven to assign a determinate truth-value to it and say, for example, that this sentence was true (in case a sea battle is actually taking place). ${ }^{2}$ Related to retrogradation is a more general concern about expressivity: the formalism should account for the intuitive meaning of different tensed constructions in natural language. This means that the the language and its semantics must be able to express the different truth-conditions of propositions like the following.

(1) There will be a sea-battle tomorrow.

(2) Laws of physics will hold tomorrow.

(3) There is a sea-battle, so it was true yesterday (but not settled) that there would be a sea-battle.

(4) The coin will come up heads. It is possible though, that it will come up tails, and then later it will come up tails again (though at that

\footnotetext{
${ }^{2}$ This is MacFarlane's determinacy intuition (see MacFarlane (2003), p. 322) as opposed to the indeterminacy intuition (future contingent sentences are neither true nor false at the moment of utterance).
} 
moment it could come up heads), and then, inevitably, still later it will come up tails yet again. ${ }^{3}$

(5) There is a sea-battle, but there could have been none.

In the next section we will present in detail Ockham's analysis (reconstructed by Prior) of the future contingents problem. In sections 3-6 we will introduce the four mentioned bivalent logical systems for solving it and discuss how they fare with respect to retrogradation and sentences like (1)-(5). A. Prior deserves the merit for having formulated two of them, in Chapter VII of his Past, Present and Future. He also deserves huge credit for introducing, in the same place, their common branching-time semantics, even though, as we will explain later, he did not grant them a major philosophical relevance.

\section{Ockham's argument}

We freely adapt Ockham's version of the argument leading from divine foreknowledge to the necessity of the future as exposed in his Tractatus de Praedestinatione (1320 ca.). Ockham carefully reconstructs the argument in order to isolate two fundamental premises of it and to eventually reject one of them. The first premise is

(P1) Necessarily, if God knew in the past that $p$, then $p$.

which is on a par with the standard epistemic principle that knowledge implies truth, formulated by the medievals as nihil scitum nisi verum. The second premise is

(P2) If it has been the case that $p$, then necessarily it has been the case that $p$,

that we can represent in a temporal language ${ }^{4}$ as

$$
P p \rightarrow \square P p
$$

\footnotetext{
${ }^{3}$ This example is taken from Belnap and Green (1994)

${ }^{4}$ Our language consists of atomic formulas $p, q, \ldots$ (to be read as "pure" present-tense sentences such as "there is a sea battle") and recursively built on Boolean operators $\neg$ ("not"), $\wedge$ ("and"),$\vee$ ("or"), $\rightarrow$ ("if - then"), the temporal operators $F$ ("it will be the case that") and $P$ ("it has been the case that") and an additional operator $\square$ to be read as "it is necessary that". We will also make use of dual operators like $G:=\neg F \neg$ ("it will always be the case that"), $H:=\neg P \neg$ ("it has always been the case that") and $\diamond:=\neg \square \neg$ ("it is possible that").
} 
and which goes under the name of the principle of necessitation of the past (PNP): quod fuit, non potest non fuisse. The kind of necessity involved here is not logical but historical necessity, or necessity per accidens as the medievals called it: what has been the case is (historically) necessary, for it is not any longer possible for it not to have been the case.

If we apply (P2) to divine foreknowledge we get as a first conclusion:

(C1) If God knew in the past that $p$, then necessarily God knew in the past that $p$.

A third premise is derived from the modal schema, $\square(p \rightarrow q) \rightarrow(\square p \rightarrow$ $\square q$ ), known as schema $\mathbf{K}$, which states that "if a conditional and its antecedent are necessary, then the consequent is also necessary". A special instance of it is

(P3) If (necessarily, if God knew that $p$, then $p$ ), then (if necessarily God knew that $p$ then necessarily $p$ ).

By Modus Ponens from (P1) and (P3) we obtain

If necessarily God knew that $p$ then necessarily $p$.

and finally, by $(\mathrm{C} 1)$ and transitivity:

If God knew that $p$, then necessarily $p$.

If $p$ is a future-tensed statement, such as "I will be sitting tomorrow" (or Ockham's favorite example "Peter will be chosen"), then future-tensed statements are necessary and determinism follows - by assuming divine foreknowledge or bivalence, which here amount to the same.

Ockham points out that this argument lies essentially on (PNP):

This argument is based on the proposition that a singular proposition true about the past is necessary. Therefore if "this is white" is true now, "this will be white was true" is necessary. Consequently, it is necessary that it happens, and it cannot come about otherwise. ${ }^{5}$

\footnotetext{
${ }^{5}$ see Ockham (1983) p. 99.
} 
Ockham's solution touches precisely on this point: he does not reject the principle but suggests a restriction of it. On the other hand, he maintains that God knows already, or from the beginning of time, which future events are going to happen. Again, since knowledge implies truth, saying that God knows that $p$ will be the case amounts to saying that it is true now that $p$ will be the case. Thus, propositions about the future already have a truth-value, even if we ignore which one, and the principle of bivalence is preserved. Indeed, throughout his Tractatus Ockham maintains that bivalence is the rationale of divine foreknowledge.

Ockham observes that one can block determinism and preserve the contingency of the future by limiting the universality of (PNP). This principle should only hold for the past and present tensed propositions which are not "equivalent" with any future tensed ones. ${ }^{6}$ Formally speaking, we should not be allowed to derive, from propositions like

$$
P p \rightarrow \square P p
$$

instances like

$$
P F p \rightarrow \square P F p
$$

by unrestricted substitution. Blocking such a free substitution and invalidating formulas like the last one is precisely what qualifies a logical solution as Ockhamist.

\section{Prior's Ockhamism}

Chapter VII of Prior (1967) offers a first axiomatization of an Ockhamist temporal logic. One of the axioms of this system is the formula $p \rightarrow \square p$, of which (PNP) is an instance. But this schema does not allow substitution of formulas containing the $F$ operator, i.e. we may derive from it instances like $P p \rightarrow \square P p$ but not $P F p \rightarrow \square P F p{ }^{7}$

In chapter VII we also find the first formulation of a sound semantics for this system: the nowadays universally adopted tree-like models for branching time. These models represent time as

\footnotetext{
${ }^{6}$ Equivalence is to be understood in the same sense in which "it was the case yesterday that I will quit smoking in two days" is equivalent with "I will quit smoking tomorrow".

${ }^{7}$ To be precise, Prior uses here a more expressive temporal language with metric operators $F_{n}$ ("it will be the case in $n$ intervals of time") and $P_{m}$ ("it was the case $m$ intervals ago"), where $n$ and $m$ are two quantifiable variables to be interpreted with (rational or real) non-negative numbers measuring intervals of time. For the sake of simplicity we will avoid using metric operators, since $F, P$ and $\square$ are sufficient for the points we need to make.
} 
... a line without beginning or end which may break up into branches as it moves from left to right (i.e. from past to future), though not the other way; so that from any point there is only one route to the left (into the past) but possibly a number of alternative routes to the right. ${ }^{8}$

From Prior's point of view this semantics is just a heuristic or pedagogical device and was not intended to constitute an alternative representation of the Ockhamist logic. ${ }^{9}$ On the contrary, the proof-theoretic approach was meant to replace and absorb the fictional representation and reification of time which is carried by a model-theoretic representation. Nonetheless, as we said, these structures have nowadays become such a universal tool that, with the risk of being injust to Prior, we will base our analysis on them. We therefore define the Ockhamist logic $\mathbf{O}$ as the set of all formulas which are valid in the class of the Ockhamist models that we are going to present.

Central to Prior's definition is the notion of a tree-like structure $\mathcal{T}$, like the one depicted in Figure 1, which is a pair $\langle T,<\rangle$, where $T$ is a set of moments $m, m^{\prime} \ldots$ and $<$ is a strict ordering relation (i.e. irreflexive, transitive and asymmetrical) over $T$, where the <-predecessors of any point $m$ are totally ordered by $<$ and where the intuitive meaning of $m<m^{\prime}$ is " $m$ precedes $m^{\prime \prime}$. A history $h$ is a maximal chain in $T$ for the relation $<$. The

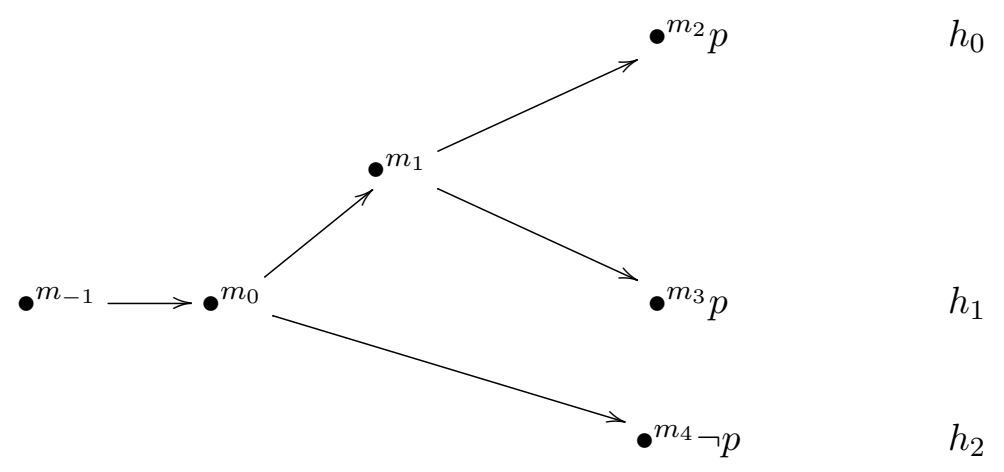

Figure 1: A model for branching time

set of histories $h_{1}, h_{2}, \ldots$ in $T$ will be denoted by $H(T)$. Given a moment

\footnotetext{
${ }^{8}$ See Prior (1967) p. 126

${ }^{9}$ This is probably one reason why Prior does not even face the question of completeness.
} 
$m, H_{m}$ will designate the set of all histories containing it. Note already that if $m<m^{\prime}$ then $H_{m^{\prime}} \subseteq H_{m}$.

A history $h$ represents a specific and well determined course of events, relative to which every proposition is true or false at $m$, including those about the future. We can formally represent that by an evaluation function $V$, which assigns a subset of $T \times H(T)$ to every propositional variable $p$ (see Fig. 1). A further requirement is that, given a moment $m, V$ does not varies with the different histories in $H_{m}$, i.e. we have

(Uniqueness) $\langle m, h\rangle \in V(p)$ if and only if for all $h^{\prime} \in H_{m},\left\langle m, h^{\prime}\right\rangle \in V(p)$

We can then define an Ockhamist model $\mathcal{M}=\langle T,<, V\rangle$ for our tensed language by extending $V$ in the following way:

$$
\begin{aligned}
& \mathcal{M},\langle m, h\rangle \models p \text { iff }\langle m, h\rangle \in V(p) \\
& \mathcal{M},\langle m, h\rangle \models \neg \phi \text { iff } \mathcal{M},\langle m, h\rangle \forall \phi \\
& \mathcal{M},\langle m, h\rangle \models \phi \wedge \psi \text { iff } \mathcal{M},\langle m, h\rangle \models \phi \text { and } \mathcal{M},\langle m, h\rangle \models \psi \\
& \mathcal{M},\langle m, h\rangle \models P \phi \text { iff } \exists m^{\prime}<m \text { such that } \mathcal{M},\left\langle m^{\prime}, h\right\rangle \models \phi \\
& \mathcal{M},\langle m, h\rangle \models F \phi \text { iff } \exists m^{\prime}>m \text { such that } \mathcal{M},\left\langle m^{\prime}, h\right\rangle \models \phi \\
& \mathcal{M},\langle m, h\rangle \models \square \phi \text { iff } \forall h^{\prime}\left(h^{\prime} \in H_{m} \Rightarrow \mathcal{M},\left\langle m, h^{\prime}\right\rangle \models \phi\right)
\end{aligned}
$$

Evaluating a future tensed proposition $F p$ w.r.t. a moment and a history amounts to checking if $p$ is satisfied "later on" in the same history. The general idea behind this is that when we talk about the future we actually pick a prima facie course of events $h$ as being the most plausible candidate among all possible futures. Historical necessity is instead equated with "truth in all histories" and, given the uniqueness condition, it is easy to check that present and past-tensed propositions (e.g. $p, P p, P P p$ etc.) are, if true, necessary.

O can easily distinguish among contingent and settled truths about the future. Indeed, contingent sentences like $F p$ ("there will be a sea-battle") may very well be true but not necessary: in our model $\left\langle m_{0}, h_{0}\right\rangle \models F p$ but $\left\langle m_{0}, h_{0}\right\rangle \models \neg \square F p$. Ockhamist semantics also respects the intuition that some sentences about the future, like (2) (see introduction), can be true in a stronger sense, i.e. also necessary, when they hold in all possible branches. This is a fortiori the case of logical tautologies $T$ : both $T$ and $\square \top$ are valid in Ockhamist models, for tautologies are true at every pair $\langle m, h\rangle$. 
It is easy to verify that (PNP) does not hold in general in this semantics and in particular, as claimed by Ockham, it fails for sentences containing a reference to the future. Indeed, as the reader may check, in the model of Figure 1 at the moment $m_{0}$ we have $\left\langle m_{0}, h_{0}\right\rangle \models P F p$ but $\left\langle m_{0}, h_{0}\right\rangle \models \neg \square P F p$. Nevertheless, in accordance with Ockham, (PNP) is valid for propositions which are "not equivalent to future-tensed ones", in our case those not containing any operator $F .{ }^{10}$

We may notice that retrogradation of truth is secured by the fact that $p \rightarrow P F p$ is valid in the Ockhamist semantics. More generally, we can easily account for sentences like (3) in section 1, which can be translated as $p \wedge P F p \wedge P \neg \square F p$ and which are true at $\left\langle m_{2}, h_{0}\right\rangle$ in our model.

Complex propositions like (4) make plural references to different possible futures at different points in the tree. Here too, Ockhamism is powerful enough to express its truth conditions. For example, (4) can be translated by the formula $F h \wedge \diamond F(t \wedge \diamond F h \wedge F(t \wedge \square F t)) .{ }^{11}$ This formula is satisfied at $\left\langle m_{0}, h_{2}\right\rangle$ by the model in Figure 2.

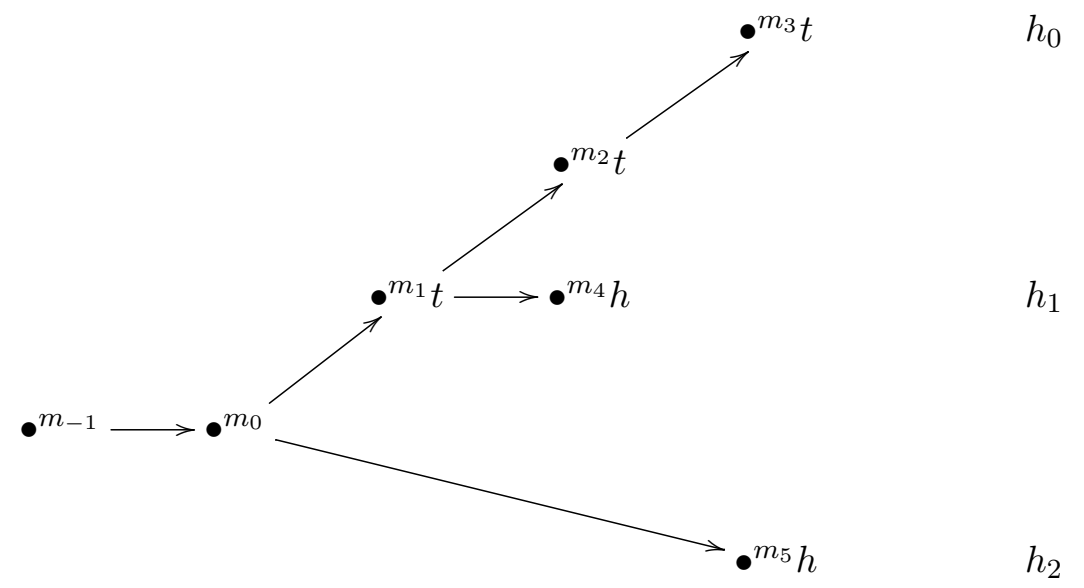

Figure 2: Heads and tails.

The intuition behind (5) of section 1 is that we should also be able to refer to this precise moment in courses of events which are, properly speaking, no more possible: this is the sense of a counterfactual with a false

\footnotetext{
${ }^{10}$ Finer-grained distinctions are induced in Prior's actual system by the use of metric operators.

${ }^{11}$ Where $h$ means "the coin lands head" and $t$ stands for "the money lands tail".
} 
antecedent. In the Ockhamist semantics this can be expressed in many cases by moving back and forth along the branches. The truth conditions of (5) can be "mimicked" by $p \wedge P \diamond F \neg p,{ }^{12}$ which is indeed satisfied in the model of Figure 1 at $\left\langle m_{2}, h_{0}\right\rangle$. Nonetheless, not all counterfactuals seem to be expressible, as (5), by simple combinations of $F, P$ and $\square$. We will come back to this point in section 6 .

To resume, Prior's Ockhamism is a very expressive framework that enables the distinction between contingent and settled propositions about the future. But there is a major philosophical objection against it, which concerns the notion of a prima facie course of events. Since, at $m$, all histories in $H_{m}$ are equally possible, it is not clear how one should be able to single out any one of them. However, according to many, when talking about future events, we need to refer to the actual future. But in this semantics (and also in Prior's view) there is no such a thing. The main problem with it seems to be its neutrality between two opposite views: one according to which there is no designated course of events, and the other which, on the contrary, allows one to refer to the actual course of events. Peirceanism and Actualism are meant to bear these opposite stands in a more radical way.

\section{The Peircean system}

Restricting (PNP) is not the only way to block arguments for determinism. As one may evince from Ockham's argument, it is also crucial that God is able to know in the past what will happen later. This is only possible if we assume that $p \rightarrow P F p$ is valid. The latter is an uncontroversial principle of minimal temporal logics, but not of Prior's Peircean logic. ${ }^{13}$ The Peircean system $\mathbf{P}$ was favored by Prior over $\mathbf{O}$ as the only one which fleshes out the intuition that the future is not "real" until it becomes present ${ }^{14}$, the only exception being represented by that parcel of the future which is already present in its causes.

Prior introduces the idea behind $\mathbf{P}$ as a variant of the traditional solution (rejecting bivalence to save indeterminism), where a different interpretation of the $F$ operator plays, in some peculiar sense, the role usually ascribed to a third truth-value or a truth-value gap. A Peircean model is easily obtained from an Ockhamist one by modifying the clause for $F$ as follows:

\footnotetext{
${ }^{12}$ This translation is not completely faithful. A metric language can better express (5) with $p \wedge P_{n} \diamond F_{n} p$.

${ }^{13}$ See Prior (1967) chap. VII p. 132.

${ }^{14}$ See Prior (1966).
} 


$$
\mathcal{M},\langle m, h\rangle \mid F \phi \text { iff } \forall h^{\prime}\left(\left(h^{\prime} \in H_{m}\right) \Rightarrow \exists m^{\prime}\left(m<m^{\prime} \wedge \mathcal{M},\left\langle m^{\prime}, h^{\prime}\right\rangle \models \phi\right)\right)
$$

Again, we identify $\mathbf{P}$ with the set of all formulas valid in the class of Peircean models. Fp now means something like "given any course of events, it will be the case that $p$ ". The intuition is that, speaking about the future, it does not make sense to pick up any prima facie designated history, since all possible futures stand on a par from the present standpoint. $F$ has now the same meaning as the expression $\square F$ in $\mathbf{O}$ : indeed $\mathbf{P}$ can be seen as a fragment of it. Thereby, $\mathbf{P}$ is also bivalent and the law of excluded middle holds also for future contingent propositions, i.e. $F p \vee \neg F p$ is valid. But, contrary to the Ockhamist semantics, $F p \vee F \neg p$ can very well fail as well as $\neg F p \rightarrow F \neg p$ (but its converse holds). ${ }^{15}$ The Peircean solution has some counterintuitive backups: future "necessary" propositions like (2) are still true, but future contingent ones like (1) are now simply false (consider the model in Figure 1 as a Peircean model). Nonetheless, one may still distinguish between necessarily false propositions, those $\phi$ s for which both $\neg F \phi$ and $F \neg \phi$ are true, and contingently false ones, those $\phi$ s for which both $\neg F \phi$ is true but $F \neg \phi$ is not.

It is easy to check that $p \rightarrow P F p$ is no more valid. Thus retrogradation of truth is undermined. In general, propositions like (3), saying that something "was going to be the case" are regarded simply as (bad) façons de parler to express the fact that something is now the case. Similar problems arise for (4) and (5) and many other examples. In general, since $\mathbf{P}$ is a proper fragment of $\mathbf{O}$, it seems that the Peircean is committed to a "deflationist" view about temporal truth, according to which many sentences we commonly express in natural language are simply misleading paraphrases.

\section{Actualism}

All along his Tractatus Ockham seems to presuppose that there is, among all possible future courses of events, a designed actual future, a sort of thin red line among all other branches ${ }^{16}$, that God already knows from all eternity. This designed history should be, contrary to Prior's claims, not only a prima facie one. Adherence to Ockham's word is not the only reason to stipulate

\footnotetext{
${ }^{15}$ It should also be noticed that the Peircean sense of "it will always be the case that" is no more expressed by the combination $\neg F \neg$, thus $G$ has to be defined as a new primitive operator by the following clause

$\mathcal{M},\langle m, h\rangle \models G \phi$ iff $\forall h^{\prime} \forall m^{\prime}\left(\left(h^{\prime} \in H_{m} \wedge m<m^{\prime}\right) \Rightarrow \mathcal{M},\left\langle m^{\prime}, h^{\prime}\right\rangle \models \phi\right)$

${ }^{16}$ This famous expression was coined by Belnap and Green (1994).
} 
such a special history. It seems that we often refer to this unique entity in order to make sense of peculiar sentences such as "Tomorrow I will quit smoking, even if all evidence speaks against that". ${ }^{17}$

There are different possible ways of defining an actualist semantics in a branching structure (see Barcellan and Zanardo (1999) and Braüner et al. (2000)), but all of them must fulfill some natural requirements. First of all, looking at sentences like (4), it seems clear that a model should not only specify a designated branch corresponding to "the true history", but also many others: one for every counterfactual moment $t$. Following Barcellan and Zanardo (1999), we define an actualist semantics on the basis of an Ockhamist model via a function $\mathcal{A}(t)$ from $T$ to $H(T)$, which picks the actual future at a moment $m$. Then we define an actual future operator $f_{A}$ with the following clause:

$$
\mathcal{M},\langle m, h\rangle \models f_{A} \phi \text { iff } \exists m^{\prime} \in \mathcal{A}(m)\left(m<m^{\prime} \wedge \mathcal{M},\left\langle m^{\prime}, \mathcal{A}(m)\right\rangle \models \phi\right)
$$

This function is supposed to respect some natural constraints, the most immediate being

TRL1 $m \in \mathcal{A}(m)$

and the second being the condition of "coherence"

TRL2 $\forall m_{1}, m_{2}\left(m_{1}<m_{2} \rightarrow \mathcal{A}\left(m_{1}\right)=\mathcal{A}\left(m_{2}\right)\right)$

According to Belnap and Green (1994), such conditions generate some serious problems of inconsistency for the actualist conception. If we put together TRL1 and TRL2 the order $<$ is forced to be linear. On the other hand, they claim, if we discard TRL2 we obtain "unreasonable results", e.g. we invalidate many natural principles such as (a) $P P \phi \rightarrow P \phi$, (b) $f_{A} f_{A} \phi \rightarrow f_{A} \phi$ and $(\mathrm{c}) \phi \rightarrow P f_{A} \phi .^{18}$

TRL2 is actually a strong coherence condition; Barcellan and Zanardo (1999) showed that we can instead reasonably opt for the weaker

TRL2* $\forall m_{1}, m_{2}\left(m_{1}<m_{2} \wedge m_{2} \in \mathcal{A}\left(m_{1}\right) \rightarrow \mathcal{A}\left(m_{1}\right)=\mathcal{A}\left(m_{2}\right)\right)$

and escape most of the "unreasonable results". They also add the further condition

TRL3 there exists an $m^{*}$ such that for all $m<m^{*}, \mathcal{A}(m)=\mathcal{A}\left(m^{*}\right)$

\footnotetext{
${ }^{17}$ For a more accurate discussion of this point see Hasle and Øhrstrom (1995) and Øhrstrom et al. (1998).

${ }^{18}$ See Belnap and Green (1994) p. 380
} 
where $\mathcal{A}\left(m^{*}\right)$ defines the unique "real" history of the model. ${ }^{19}$ It is possible to check that this definition preserves many temporal laws such as (a) and (b). The formula (c) $\phi \rightarrow P f_{A} \phi$ is not valid instead - as an example, consider the failure of $t \rightarrow P f_{A} t$ at $m_{1}$ in the model in figure 3 - but is nonetheless satisfied at any moment of $\mathcal{A}\left(\mathrm{m}^{*}\right)$. An additional problem for

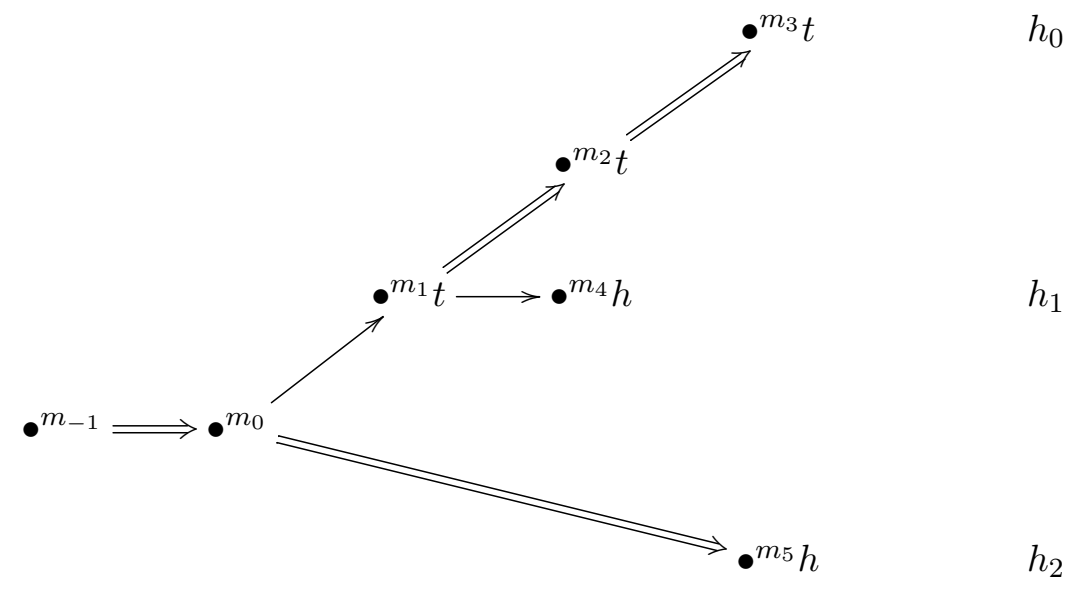

Figure 3: Failure of $\phi \rightarrow P f_{A} \phi$. Double arrows indicate each moment's actual future.

this semantics is that it cannot properly block (PNP) for, as one may easily check,

$$
f_{A} \phi \rightarrow \square f_{A} \phi
$$

is a valid formula, as well as its converse. From this point of view, actualism is not completely Ockhamist. In order to make (PNP) fail and express (1)-(5) one should enrich the language with other future tense operators. ${ }^{20}$

To summarize, the major "logical" inconvenience of the actualist operator $f_{A}$ is that when we combine it with $P$ and $\square$ many "natural" principles seem to fail and we have to recur to other future operators to adjust them. But it is fair to notice that failures of "intuitive" principles are not specific of $f_{A}$ and that they at least do not seem to lead to an "inconsistency" of the actualist conception. ${ }^{21}$ From a more metaphysical standpoint, the most common objection to actualism, in this or other forms, is that it involves a

\footnotetext{
${ }^{19}$ For a proof of uniqueness see Barcellan and Zanardo (1999) p. 5.

${ }^{20}$ Barcellan and Zanardo use the peircean operators of section 2 as primitives.

${ }^{21}$ For a more articulated defence of actualism see Øhrstrom (2009).
} 
commitment to facts "that do not supervene upon any physical, chemical or psychological states of affairs" (Belnap and Green (1994)).

\section{$6 \mathrm{~T} \times \mathrm{W}$ semantics}

Branching time semantics are not the only possible "technical" solution for preserving future contingency and bivalence. Another option is represented by $T \times W$ semantics, introduced in Thomason (1984). ${ }^{22}$ Whereas branchingtime is based on the idea of overlapping histories, $T \times W$ starts from the intuition of there being a plurality of separated possible courses of events (or worlds) which may have "equivalent" past histories up to a point and diverge afterwards. ${ }^{23}$ The models of figure 3 represent this difference.

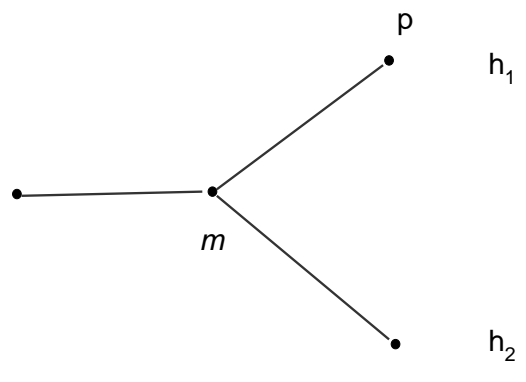

(a) a tree-like model

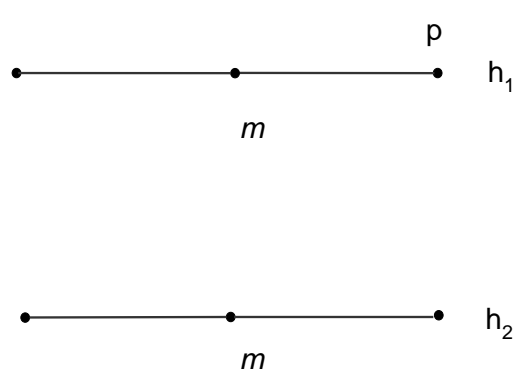

(b) a $T \times W$ model

Figure 4: Ockhamist models

For a formal definition, we need a set $T$ of moments, an irreflexive linear order $<$ on it, a set $W$ of possible worlds and a family $\left\{\sim_{t} \mid t \in T\right\}$ of equivalence relations among them, intuitively denoting sameness up to a certain point in time $t$. A frame is a tuple $\left\langle T \times W,<,\left\{\sim_{t}\right\}_{t \in T}\right\rangle$ where

- $T \times W$ is the set of $\langle t, w\rangle$ such that $t \in T$ and $w \in W$

- for all $t \in T, \sim_{t}$ is an equivalence relation

- for all $t^{\prime} \in T$, if $w \sim_{t} w^{\prime}$ and $t^{\prime}<t$ then $w \sim_{t^{\prime}} w^{\prime}$

\footnotetext{
${ }^{22}$ Complete logical systems for this semantics have been formulated later by von Kutschera (1997) and Di Maio and Zanardo (1998).

${ }^{23}$ For the notions of overlap and divergence see the famous Lewis (1986) pp. 198-209.
} 
Given a valuation $V$, assigning to every $p$ a subset of $W \times T$, a model is obtained by expanding $V$ to a satisfaction relation in the usual way for Boolean and temporal operators (e.g. $\langle t, w\rangle \models F \phi$ iff for some $t^{\prime}>t$, $\left.\left\langle t^{\prime}, w\right\rangle \models \phi\right)$ and defining the $\square$-clause as:

$$
\langle t, w\rangle \models \square \phi \text { iff for all } w^{\prime} \text { such that } w \sim_{t} w^{\prime},\left\langle t, w^{\prime}\right\rangle \models \phi
$$

Necessity at $\langle t, w\rangle$ means truth at the "same" moment in all other equivalent histories. We get the Ockhamist notion of historical necessity by an adequate specification of $\sim_{t}$ as "sharing the same past up to $t$ " i.e.

$w \sim_{t} w^{\prime}$ iff for all $t^{\prime} \leq t,\left\langle t^{\prime}, w^{\prime}\right\rangle$ and $\left\langle t^{\prime}, w\right\rangle$ satisfy the same propositional letters.

It is relevant to notice that under some specific conditions a branching Ockhamist model can be tranformed into a $T \times W$-model in a truth preserving way. This happens when we have a synchronized tree, i.e. a tree whose branches are all isomorphic. ${ }^{24}$ Under this condition the $T \times W$ semantics is at least as expressive as the Ockhamist semantics of Section 3, i.e. we can account in the same way for the truth conditions of (1)-(5), and even more. ${ }^{25}$ Indeed, in $T \times W$ necessity operators are defined via a more arbitrary equivalence relation among histories, which does not force the uniqueness condition (see Section 3). Therefore, one is free to define new necessity and possibility operators by relaxing or making more accurate the equivalence relations. Relaxing the equivalence relation allows to quantify over histories that diverge even before a given moment $m$. By this means, it is possible

\footnotetext{
${ }^{24}$ More precisely, a synchronized tree is a tree-like structure where it is possible to define a partition $I$ (the "instants") of the set $T$ that satisfies the following conditions (see also Wölfl (2002)):
}

(a) For every $i \in I$ and every $h \in H(T)$ there is exactly one $m_{i, h} \in T$ with $m_{i, h} \in i \cap h$

(b) For all $i, i^{\prime} \in I$ and all $h, h^{\prime} \in H(T)$, from $m_{i, h}<m_{i^{\prime}, h}$ it follows that $m_{i, h^{\prime}}<m_{i^{\prime}, h^{\prime}}$

Given a synchronized Ockhamist model $\mathcal{T}=\langle T,\langle, V\rangle$ we can define a $T \times W$ model $\mathcal{T}^{\prime}=\left\langle T^{\prime} \times W^{\prime},<^{\prime},\left\{\sim_{t}\right\}_{t \in T}, V^{\prime}\right\rangle$ by taking:

- $T^{\prime}=I$ and $W^{\prime}=H(T)$

- $i<^{\prime} i^{\prime}$ iff $m_{i, h}<m_{i^{\prime}, h}$ for some $h \in H(T)$

- $h \sim_{i} h^{\prime}$ iff $m_{i, h}=m_{i, h^{\prime}}$

- $\langle i, h\rangle \in V^{\prime}(p)$ iff $\left\langle m_{i, h}, h\right\rangle \in V(p)$

and it is straightforward to check that $\mathcal{T},\langle m, h\rangle \models \phi$ if and only if $\mathcal{T}^{\prime},\langle m, h\rangle \models \phi$.

${ }^{25}$ The situation is more complex if the tree is not synchronized. For an accurate study of the relationships between branching-time semantics and $T \times W$ see Wölfl (2002). 
to handle propositions like "for all that I know it could have been raining last night", where the construction "for all that I know ..." is to be read as an epistemic possibility operator. Here, indeed, we are driven to consider as epistemic alternatives more histories than those which share the same past. ${ }^{26}$ The case of counterfactuals presents analogous features. ${ }^{27}$

\section{Conclusions}

We have presented four logical systems which deal with a perennial philosophical problem: the problem of future contingents. Apart from tackling the problem in a rigorous way, the four logical approaches have the same model-theoretical format. This makes the solutions comparable and allows us to see what are the gains and losses in terms of expressivity, the relation between future contingents and the principle of bivalence, and the metaphysical commitments we make.

The system $T \times W$ has at least the same expressive power as the Ockhamist semantics, but it has received scarce attention or has even been fiercely opposed. Thomason himself dismissed it in the very same paper in which he introduced it Thomason (1984). Most of the reasons for this attitude are grounded in metaphysical considerations. Whereas branching time is regarded as a misleading but almost an adequate representation of McTaggart's A-series conception, $T \times W$ is instead associated with the Bseries conception and seems to commit to a reification of time. ${ }^{28}$ Moreover, quantification over non actual and non overlapping histories is seen by many

\footnotetext{
${ }^{26}$ See also Iacona (2009).

${ }^{27}$ The same "redefinitions" of necessity and possibility operators can of course be carried out, in principle, also in an Ockhamist model. However, this goes against one of the philosophical motivations behind the branching time semantics, according to which all tensed constructions ought to be expressed with reference to points in time that are connected to the present point of evaluation - by some (back and forth) path over the temporal tree. This requirement may be too restrictive when we need to consider, e.g., fictional alternatives or histories diverging in the far past.

${ }^{28}$ The notions of A-series and B-series were introduced by McTaggart (1908). The Aseries conception of time, also called the dynamic view, resumes the way we experience time by being "in a flux" and opens up to presentism - a view that McTaggart himself did not endorse - where only the (constantly changing) "now" properly exists. According to this conception past, present, and future tenses are primitive concepts for referring to events in time. Other temporal concepts such as instants in time and the earlier-later relation between them, are to be derived from the formers. On the other hand, according to the B-series conception - which accounts for a "bird-eye view" of time and according to which the entire series of instants exists - instants and their earlier-later relation are the primitive concepts and tenses are derived from them.
} 
as an additional commitment to modal realism (i.e. the philosophical thesis that non actual worlds are real or exist on a par with the actual one). To many, overlap seems more faithful to an intuitive notion of causality: at any moment $m$ there is just one past that we cannot change or influence and many possible futures we can "act upon" and "decide which one to take". In $T \times W$, at any point, there is just one future; contingency and causal influence on the future seem to be definable only in terms of a counterfactual dependence $^{29}$, i.e. in terms of what would happen if the actual course of events were different.

In response to the criticisms against $T \times W$ one may point out that it is not clear how the choice of a particular semantics should commit us to a certain ontology. Additionally, it does not seem that other bivalent approaches like Ockhamism and Actualism are safe from these problems: if bivalence holds and truth is relative to a particular course of events then we are just one step far from admitting that other courses of events are fictional ones, and that the metaphor of branching seems just an unsuccessful compromise. $T \times W$ keeps the order of truth and the order of causality on two separate plans. Peirceanism, with a radically different definition of truth for future tensed propositions, seems to be the only radical alternative. However, defenders of Peirceanism face at least two burdens: they should deal with a less expressive language and have to find a justification for the strange asymmetry which makes it that future contingents are just false. Non-bivalent approaches admitting truth-value gaps for future contingents, as the one defined in Thomason (1970) and which Prior hoped for ${ }^{30}$, seem to be the only possible way to fully preserve symmetry between truth and falsity.

\section{References and recommended readings}

B. Barcellan and A. Zanardo. Actual futures in peircean branching-time logic, 1999. URL citeseer.ist.psu.edu/326930.html.

N. Belnap and M. Green. Indeterminism and the thin red line. In J. Tomberlin, editor, Philosophical Perspectives 8: Logic and Language, pages 217244. Atascadero, 1994.

T. Braüner, P. Øhrstrom, and F. Hasle. Determinism and the Origins of

\footnotetext{
${ }^{29}$ See Lewis (1979).

${ }^{30}$ See Prior (1967) p. 137.
} 
Temporal Logic. In H. Barringer, M. Fisher, D. Gabbay, and G. Gough, editors, Advances in Temporal Logics, pages 185-206. Kluwer, 2000.

M. Di Maio and A. Zanardo. A Gabbay-Rule Free Axiomatization of $\mathrm{T} \times \mathrm{W}$ Validity. Journal of Philosophical Logic, 27(5):435-487, 1998.

P. Hasle and P. Øhrstrom. Temporal Logic from Ancient Ideas to Artificial Intelligence. Kluwer, 1995.

A. Iacona. Commentary: Combinations of tense and Modality by R. Thomason. Humana.Mente, 8:185-190, 2009.

D. Lewis. Counterfactual Dependence and Time's Arrow. Nous, 13:455-76, 1979.

D. Lewis. On the Plurality of Worlds. Blackwell, Oxford, 1986.

J. MacFarlane. Future contingents and relative truth. The Philosophical Quarterly, 53:321-336, 2003.

J. M. E. McTaggart. The unreality of time. Mind, 187:457-474, 1908.

W. Ockham. Predestination, God's Foreknowledge, and Future Contingents. Hackett, Indianapolis, 1983.

P. Øhrstrom. In defence of the thin red line: a case for ockhamism. Humana.mente, 8:17-32, 2009.

P. Øhrstrom, P. Hasle, and T. Braüner. Ockhamistic logics and true futures of counterfactual moments. In Proceedings of the Fifth International Workshop on Temporal Representation and Reasoning (TIME-98), volume 18, 1998.

A. N. Prior. Postulates for tense-logic. American Philsophical Quarterly, 3, 1966.

A. N. Prior. Past, Present and Future. Oxford University Press, Oxford, 1967.

R. Thomason. Indeterminist time and truth-value gaps. Theoria, 36(3): 264-281, 1970.

R. H. Thomason. Combinations of tense and modality. In F. G. D. Gabbay, editor, Handbook of Philosophical Logic, pages 205-234. Kluwer Academic Publisher, Dordrecht, 1984. 
F. von Kutschera. $T \times W$ Completeness. Journal of Philosophical Logic, 26 : 241-250, 1997.

S. Wölfl. Propositional Q-Logic. Journal of Philosophical Logic, 31:387-414, 2002 . 